\title{
Nitric Oxide Elevation in Polytrauma is Driven by Oxygen Radicals
}

\author{
E. BEITL ${ }^{1}$, A. BAŇASOVÁ ${ }^{2,3}$, D. MIKOVÁ ${ }^{2}$, V. HAMPL ${ }^{2}$ \\ ${ }^{1}$ First Orthopaedic Clinic, First Faculty of Medicine, Charles University, Prague, Czech Republic, \\ ${ }^{2}$ Department of Physiology, Second Faculty of Medicine, Charles University, Prague, Czech \\ Republic, ${ }^{3}$ Department of Anaesthesiology and Intensive Care Medicine, Second Faculty of \\ Medicine, Charles University, Prague, Czech Republic
}

Received March 21, 2017

Accepted September 6, 2017

\section{Summary}

A common problem in management of polytrauma a simultaneous injury to more than one organ or organ system, at least one of them lethal without intervention - is a discrepancy between a relatively good initial state and a serious subsequent development. Since nitric oxide (NO) is produced in high quantities during tissue injury, we assumed that serum levels of NO (and its oxidation products, NOx) might serve as a prognostic marker of polytrauma severity. However, we found recently that NOx was increased in polytrauma, but not in the most severe cases. The present study was undertaken to test the hypothesis that serum NOx is reduced in severe polytrauma by concomitant overproduction of reactive oxygen species (ROS). Polytrauma was induced in rats under anesthesia by bilateral fracture of femurs and tibiae plus incision of the right liver lobe through laparotomy. Serum NOx was measured by chemiluminescence after hot acidic reduction. The role of ROS was assessed by treatment with an antioxidant, N-acetyl-L-cysteine (NAC). Experimental polytrauma elevated NOx from $11.0 \pm 0.7$ to $23.8 \pm 4.5 \mathrm{ppb}$. This was completely prevented by NAC treatment $(9.1 \pm 2.2 \mathrm{ppb})$. Serum NOx is elevated in severe polytrauma, and this is not reduced by ROS. On the contrary, ROS are necessary for the NOx elevation, probably because ROS produced by inflammatory cells activated by the polytrauma induce massive NO production.

\section{Key words}

Nitric oxide $\bullet$ Polytrauma $\bullet$ Shock $\bullet \mathrm{N}$-acetyl-L-cysteine $\bullet$ Reactive oxygen species $\bullet$ Antioxidant

\section{Corresponding author}

A. Baňasová, Department of Physiology, Second Faculty of Medicine, Charles University, Prague, Plzeňská 221,
15000 Prague 5, Czech Republic. E-mail: alena.banasova@lfmotol.cuni.cz

\section{Introduction}

Polytrauma is defined as a simultaneous injury to more than one organ or organ systems, where at least one of those injuries is of such a degree or nature that, without urgent treatment, can cause death. Patients with polytrauma are at high risk of developing shock that can quickly lead to death. Even if shock is managed successfully, another serious risk is the development of systemic inflammatory response syndrome (SIRS), frequently followed by multiorgan failure (Keel et al. 2005). A common clinical problem in polytrauma is a discrepancy between a relatively satisfactory initial clinical state of the patient on one hand and a serious subsequent development on the other. Therefore, an early marker of the severity of polytrauma with a predictive value is desirable.

Nitric oxide (NO) is produced in high quantities during tissue injury (Pacher et al. 2007) and this overproduction contributes to the development of shock. It is thus reasonable to expect that plasma levels of stable NO forms on admission might represent such a marker. In aqueous milieu, NO is rapidly oxidized by oxygen to nitrites and, in the presence of heme proteins, to nitrates (Moncada et al. 1993). Therefore, sum of plasma concentrations of NO and its oxidation products, NOx, has to be used instead of just NO concentration (Archer $e t$ al. 1994, Archer et al. 1995).

In line with this reasoning, we found in a recent study that plasma NOx concentration on admission correlated positively with the severity of subsequent 
clinical course of patients with polytrauma (Beitl et al. 2016). Surprisingly, though, this was not true for the most critically ill patients - those who needed resuscitation or subsequently died. Their plasma NOx on admission was similarly low as in healthy controls (Beitl et al. 2016).

One possible explanation of this paradox could be a rapid inactivation of NO by its reaction with superoxide, a radical that is produced at a high rate during inflammatory response to tissue injury (Aller et al. 2004). If this explanation is valid, then the low plasma NOx in extreme polytrauma should not occur if the superoxideNO interaction is prevented (e.g. by amplified superoxide scavenging).

To test this hypothesis, we utilized a rat model of polytrauma and measured plasma NOx with and without treatment with $\mathrm{N}$-acetyl-L-cysteine (NAC). NAC is a precursor of glutathione that, in turn, is one of the main defenses of the organism against oxidative stress (Halliwell 2012, Koekkoek et al. 2016, Zhang et al. 2011). Our laboratory had previously used NAC to affect pathological processes in the lungs (Lachmanová et al. 2005).

\section{Methods}

Three groups of adult male Wistar rats (body weight $444-570 \mathrm{~g}$ with no differences among the groups) were used with the permission of the Ethical Committee of our institution, in accordance with EU standards. All procedures were performed under deep anesthesia with IP ketamine $(100 \mathrm{mg} / \mathrm{kg})+$ xylazine $(16 \mathrm{mg} / \mathrm{kg})$. In one group (CA, $\mathrm{n}=6$ ), blood samples for NOx (and hematocrit) determination were drawn from a cannulated carotid artery before and $12-20 \mathrm{~min}$ after inflicting experimental polytrauma (pre-PT and post-PT measurement, respectively). The catheter was also used to follow the mean systemic arterial blood pressure (SBP). In another group, the procedure was identical, but an i.p. injection of NAC aqueous solution $(400 \mathrm{mg} / \mathrm{kg})$ (Akbay et al. 1999) was given concurrently with the induction of anesthesia $(\mathrm{CA}+\mathrm{NAC}, \mathrm{n}=6)$. Since the process of carotid artery cannulation itself represents a degree of trauma, another group was used in which this procedure was avoided. Blood samples in this group $(\mathrm{LV}, \mathrm{n}=6)$ were obtained under the same anesthesia by a quick puncture of the left ventricle across the chest wall.

Polytrauma was modeled under anesthesia by bilateral fracture of femurs and tibiae (Ban et al. 2008) plus incision of the right liver lobe through laparotomy. This represents a severe polytrauma (in humans with similar injuries, the ISS score would be above 25). The ensuing blood loss resulted into gradual SBP decline. The post-PT blood sample for NOx determination was taken when the SBP dropped to one half of its initial value.

Serum NOx concentration was measured by chemiluminescence NO analyzer (Sievers NOA 270) after hot acidic reduction, as described previously (Beitl et al. 2016, Hampl et al. 1996), and expressed as parts per billion (ppb) of NO in the stripping gas.

The data were evaluated statistically using the GraphPad Prism 6 software. Unpaired t-test, one-way ANOVA followed by Tukey's multiple comparisons post hoc test or two-way ANOVA were used as appropriate, with $\mathrm{P}<0.05$ considered significant. The results are presented as means \pm S.E.M.

\section{Results}

In the LV group, serum NOx concentration was $11.0 \pm 0.7 \mathrm{ppb}$. There was a tendency for the carotid artery cannulation procedure to increase NOx (to $15.6 \pm 1.6 \mathrm{ppb}$ in the CA group pre-PT) that, however, did not reach statistical significance. The experimental polytrauma caused further, this time significant, rise in NOx (to $23.8 \pm 4.5 \mathrm{ppb}$; Fig. 1). NAC administration at the time of inducing anesthesia resulted in NOx concentration pre-PT that was significantly lower than that without NAC $(9.1 \pm 2.2 \mathrm{ppb})$. NAC prevented any increase in NOx after polytrauma (Fig. 1).

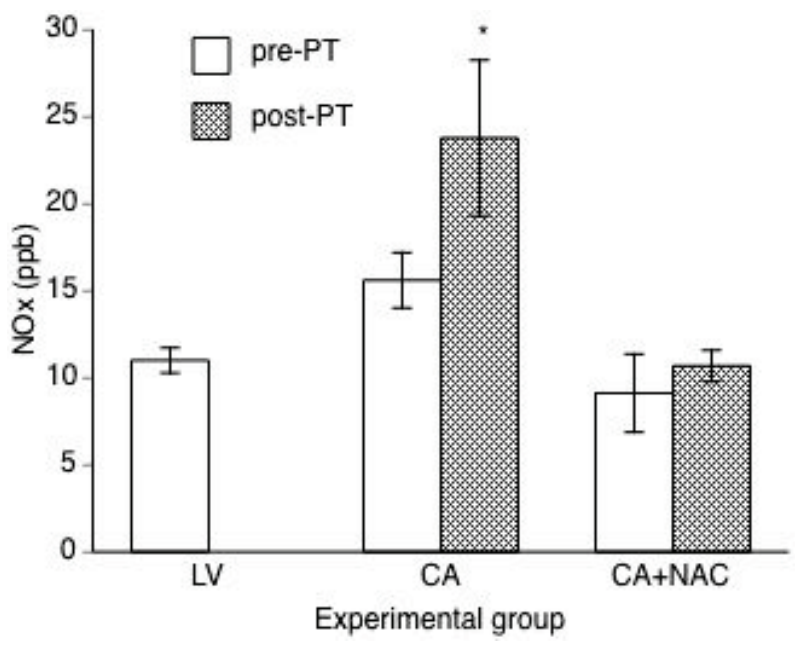

Fig. 1. $\mathrm{N}$-acetyl-L-cysteine (NAC) prevents an increase in serum NOx concentrations caused by experimental polytrauma. $\mathrm{LV}=$ the group in which blood samples were obtained by left heart ventricle puncture; $\mathrm{CA}=$ the group in which blood samples were obtained from cannulated carotid artery; $C A+N A C=$ the group in which blood samples were obtained from cannulated carotid artery after treatment with NAC; pre-PT = measurement obtained before inflicting experimental polytrauma; post-PT $=$ measurement obtained when systemic blood pressure fell to $50 \%$ of its initial value after inflicting experimental polytrauma. $* \mathrm{P}<0.05$. 
Administration of NAC did not affect mean SBP pre-PT $(68.5 \pm 3.8 \mathrm{~mm} \mathrm{Hg}$ in $\mathrm{CA}, 73.5 \pm 5.0 \mathrm{~mm} \mathrm{Hg}$ in $\mathrm{CA}+\mathrm{NAC}, \quad \mathrm{P}>0.05), \quad$ nor post-PT $(28.8 \pm 4.6$ vs. $19.3 \pm 0.7 \mathrm{~mm} \mathrm{Hg}, \mathrm{P}>0.05)$. Similarly, hematocrit was not affected by NAC pre-PT $(42.5 \pm 0.9 \%$ in CA, $41.3 \pm 0.7$ in $\mathrm{CA}+\mathrm{NAC}, \quad \mathrm{P}>0.05)$ nor post-PT $(19.2 \pm 0.5 \quad$ vs. $14.3 \pm 2.3 \%, \mathrm{P}>0.05)$.

\section{Discussion}

The main finding of this study is that augmentation of antioxidant defense by NAC administration prevents serum NOx increase caused by experimental polytrauma. In addition, this experiment in rats confirms our previous finding in human patients that serum NOx is a suitable candidate for a marker of the severity of polytrauma clinical course. Our observation that the procedure of carotid artery cannulation did not result in an increase in serum NOx that would be statistically significant corresponds to our previous finding in humans that only more severe, but not minor, injuries cause NOx elevation (Beitl et al. 2016). In terms of practical utility, this is advantageous - serum NOx seems to be a selective marker of only a serious, but not minor, injury. This - together with the significant elevation of NOx after experimental polytrauma confirms the selectivity of this marker for just serious injuries.

The present study in rats did not replicate our previous finding in human patients that serum NOx increases only up to quite severe polytrauma, but the most serious cases display normal NOx levels (Beitl et al. 2016). In fact, our experimental rats were in a serious condition after the polytrauma - their mean SBP fell by about one-half at the time of the post-PT NOx measurement, as did their hematocrit. The reason for this discrepancy cannot be revealed from our data, but it is possible that the differences in methodology might be responsible. In human patients, NOx levels had predictive value only when measured on admission to the trauma center, but not later (Beitl et al. 2016). In the experimental rats, the post-PT values were measured only after the animals proceeded to a critical condition. Alternatively, the differences in treatment are likely to contribute to the observed discrepancy. While the human patients with severe polytrauma received massive supporting therapy, the rats were left with no intervention.

Treatment with NAC completely prevented the increase in NOx after polytrauma. This result very clearly refutes the hypothesis that increased superoxide levels are responsible for the reduction of NOx observed in human patients with extremely severe polytrauma. If it were so, NAC treatment should potentiate, rather than reduce NOx. Our present results prove that the causality is the other way around - ROS are necessary for the elevation of NOx in polytrauma. The most likely explanation is that superoxide (and/or other ROS) produced by inflammatory cells activated by the polytrauma induce massive NO production. One likely mechanism involves stabilization of hypoxia-inducible factor(s) (HIF) by ROS (Niecknig et al. 2012, Pan et al. 2007). HIF is well known to regulate vascular endothelial growth factor (VEGF) (Forsythe et al. 1996) that, in turn, induces and upregulates NO synthase (Kroll et al. 1998, Shen et al. 1999). VEGF is upregulated in polytrauma (Grad et al. 1998, Vajtr et al. 2014). The NO production must be high, as the interaction with superoxide appears insufficient to offset this effect. The most likely source of greatly elevated NO production is the inducible isoform of the NO synthase. Most of the cells that express this enzyme are sensitive to oxidative stress (Preiser 2012).

NO participates in the maintenance of SBP (Hampl et al. 1993). Thus, reduced NOx after NAC treatment in polytrauma might be expected to help prevent the drop in SBP. However, We did not observe any significant effect on SBP post-PT caused by NAC. It is possible more effect could be seen with more prolonged duration, or factors other than NO play considerably larger role in severe hypotension.

An important practical question is whether our results with NAC can have some practical utility. Serum NOx concentration can either be just a marker of injury, or it can, in addition, have a pathogenetic role in the consequences of polytrauma. If the latter is true, then augmentation of the antioxidant defense, for example by NAC (a substance already used clinically), can improve the prognosis of patients with severe polytrauma. This is quite likely, inasmuch high production of the highly reactive NO are well known to damage proteins, lipids and DNA (Pacher et al. 2007). Further research of this issue is warranted.

In conclusion, we have shown that experimental polytrauma in rats causes elevation in serum NOx concentration that is prevented by antioxidant treatment with NAC. This implies that ROS production is necessary for the NOx to rise after polytrauma. 


\section{Conflict of Interest}

There is no conflict of interest.

\section{Acknowledgements}

Supported by Grant Agency of the Czech Republic grant number GA13-01710S.

\section{References}

AKBAY A, CINAR K, UZUNALIMOGLU O, ERANIL S, YURDAYDIN C, BOZKAYA H, BOZDAYI M: Serum cytotoxin and oxidant stress markers in $\mathrm{N}$-acetylcysteine treated thioacetamide hepatotoxicity of rats. Hum Exp Toxicol 18: 669-676, 1999.

ALLER MA, ARIAS JL, ARIAS J: Post-traumatic inflammatory response: perhaps a succession of phases with a nutritional purpose. Med Hypotheses 63: 42-46, 2004.

ARCHER S, HAMPL V, MCKENZIE Z, NELSON D, HUANG J, SHULTZ P, WEIR EK: Role of endothelial-derived nitric oxide in normal and hypertensive pulmonary vasculature. Semin Respir Med 15: 179-189, 1994.

ARCHER SL, SHULTZ PJ, WARREN JB, HAMPL V, DEMASTER EG: Preparation of standards and measurement of nitric oxide, nitroxyl, and related oxidation products. Methods 7: 21-34, 1995.

BAN Y, SHEN H, LI TS: An experimental study of a rat model with MODS as a result of trauma induced infection. (In Chinese) Zhongguo Wei Zhong Bing Ji Jiu Yi Xue 20: 41-44, 2008.

BEITL E, BAŇASOVÁ A, MIKOVÁ D, HAMPL V: Nitric oxide as an indicator for severity of injury in polytrauma. Bratisl Med J 117: 217-220, 2016.

FORSYTHE JA, JIANG BH, IYER NV, AGANI F, LEUNG SW, KOOS RD, SEMENZA GL: Activation of vascular endothelial growth factor gene transcription by hypoxia-inducible factor 1. Mol Cell Biol 16: 4604-4613, 1996.

GRAD S, ERTEL W, KEEL M, INFANGER M, VONDERSCHMITT DJ, MALY FE: Strongly enhanced serum levels of vascular endothelial growth factor (VEGF) after polytrauma and burn. Clin Chem Lab Med 36: 379-383, 1998.

HALLIWELL B: Free radicals and antioxidants: updating a personal view. Nutr Rev 70: 257-265, 2012.

HAMPL V, ARCHER SL, NELSON DP, WEIR EK: Chronic EDRF inhibition and hypoxia: effects on pulmonary circulation and systemic blood pressure. J Appl Physiol 75: 1748-1757, 1993.

KEEL M, TRENTZ O: Pathophysiology of polytrauma. Injury 36: 691-709, 2005.

KOEKKOEK WA, VAN ZANTEN AR: Antioxidant vitamins and trace elements in critical illness. Nutr Clin Pract 31: 457-474, 2016.

KROLL J, WALTENBERGER J: VEGF-A induces expression of eNOS and iNOS in endothelial cells via VEGF receptor-2 (KDR). Biochem Biophys Res Commun 252: 743-746, 1998.

LACHMANOVÁ V, HNILIČKOVÁ O, POVÝŠILOVÁ V, HAMPL V, HERGET J: N-acetylcysteine inhibits hypoxic pulmonary hypertension most effectively in the initial phase of chronic hypoxia. Life Sci 77: 175-182, 2005.

MONCADA S, HIGGS A: The L-arginine-nitric oxide pathway. N Engl J Med 329: 2002-2012, 1993.

NIECKNIG H, TUG S, REYES BD, KIRSCH M, FANDREY J, BERCHNER-PFANNSCHMIDT U: Role of reactive oxygen species in the regulation of HIF-1 by prolyl hydroxylase 2 under mild hypoxia. Free Rad Res 46 : 705-717, 2012.

PACHER P, BECKMAN JS, LIAUDET L: Nitric oxide and peroxynitrite in health and disease. Physiol Rev 87: 315-424, 2007.

PAN Y, MANSFIELD KD, BERTOZZI CC, RUDENKO V, CHAN DA, GIACCIA AJ, SIMON MC: Multiple factors affecting cellular redox status and energy metabolism modulate hypoxia-inducible factor prolyl hydroxylase activity in vivo and in vitro. Mol Cell Biol 27: 912-925, 2007.

PREISER JC: Oxidative stress. JPEN J Parenter Enteral Nutr 36: 147-154, 2012.

SHEN BQ, LEE DY, ZIONCHECK TF: Vascular endothelial growth factor governs endothelial nitric-oxide synthase expression via a KDR/Flk-1 receptor and a protein kinase $\mathrm{C}$ signaling pathway. J Biol Chem 274: 33057-33063, 1999. 
VAJTR D, SPRINGER D, STANEK L, BENADA O, SAMAL F, BERAN M, STREJC P, PRUSA R, ZIMA T: Pathomorphology of inflammatory response following traumatic brain injury, serum values of interleukins, and gene polymorphisms. Soud Lek 59: 40-47, 2014.

ZHANG F, LAU SS, MONKS TJ: The cytoprotective effect of N-acetyl-L-cysteine against ROS-induced cytotoxicity is independent of its ability to enhance glutathione synthesis. Toxicol Sci 120: 87-97, 2011. 